\title{
All-Angle Broadband Negative Refraction of Metal Waveguide Arrays in the Visible Range: Theoretical Analysis and Numerical Demonstration
}

\author{
Xiebin Fan, ${ }^{1}$ Guo Ping Wang, ${ }^{1, *}$ Jeffrey Chi Wai Lee, ${ }^{2}$ and C. T. Chan ${ }^{2}$ \\ ${ }^{1}$ Key Laboratory of Acoustic and Photonic Materials and Devices, Ministry of Education, and Department of Physics, \\ Wuhan University, Wuhan 430072, China \\ ${ }^{2}$ Department of Physics, Hong Kong University of Science and Technology, Clear Water Bay, Kowloon, Hong Kong, China
}

(Received 20 May 2006; published 14 August 2006)

\begin{abstract}
In this Letter, we introduce a simple metal waveguide array for realizing all-angle wide frequency bandwidth negative refraction from the visible to infrared frequencies. Theoretical analysis from the rigorous coupled-wave theory reveals that the negative coupling constant resulting from the anomalous coupling of guided surface plasmon polariton modes contributes to the negative refraction. The analytical results are confirmed by finite-difference time-domain numerical simulations. Our result provides an alternative way to construct robust all-angle negative refractive materials operating in a wide range of frequency from the near-infrared to the visible range.
\end{abstract}

PACS numbers: 42.82.Et, 42.25.-p, 73.20.Mf, 78.67.-n

The negative-refraction (NR) effect [1] has currently attracted considerable interest because it underlays the foundation of a variety of new potential applications [2] and the possibility of investigating extraordinary electromagnetic wave propagation phenomena [3]. It has been demonstrated theoretically and experimentally that both left-handed metamaterials [4-7] and photonic crystals [8-15] can achieve NR from microwave to near-infrared frequencies. Furthermore, all-angle NR (AANR) for lens imaging at microwave frequencies by metamaterials $[16,17]$ and at optical frequencies by photonic crystals $[18,19]$ has also been demonstrated recently, but the operating bandwidth is still limited in a narrower frequency range [16-19], and the realization of broadband AANR at an optical frequency, especially in the visible region, is still a challenge.

Generally speaking, a periodic structure is capable of producing NR for electromagnetic waves. For example, a simple dielectric waveguide array can bend light in the direction opposite to that observed in common media at a limited incident angle within a narrow frequency bandwidth [20,21]. However, less attention was paid to its metallic counterparts, especially the nanoscale ones [22], until a very recent exploration that nanoscale metal waveguide arrays (MWGAs) show an interesting lens effect at a fixed optical wavelength [23]. In this Letter, we will reveal that nanoscale MWGAs can actually achieve AANR over a wide frequency bandwidth from visible to infrared ranges. We show the NR effect by the coupled-wave theory and further establish the effect by finite-difference timedomain (FDTD) numerical simulations. Further analysis on the conditions for AANR implies that MWGAs are suitable for visible wavelength operation in a wide bandwidth. We attribute the NR effect to coupling of propagating surface plasmon polaritons (SPPs) among the adjacent guides.

Our work begins with the analysis of a simple discrete system containing two adjacent two-dimensional (2D) metal waveguides by a rigorous field analysis approach, where linear coupling of propagating SPPs in the guides is considered. The structure is schematically drawn in Fig. 1(a); $h$ is the guide width and $d$ is the thickness of the metal film. $\varepsilon_{1}(>0)$ and $\varepsilon_{2}(<0)$ represent the relative permittivities of dielectric material in guide regions and metal material, respectively. The transverse magnetic field distribution $H_{y}$ of SPP modes in the waveguides (along the $y$ axis) can be written as:

$$
H_{y}= \begin{cases}A_{0} e^{-p(x-h-d / 2)} & x>h+d / 2 \\ B_{0} e^{k(x-h-d / 2)}+C_{0} e^{-k(x-d / 2)} & h+d / 2>x>d / 2 \\ D_{0} e^{p(x-d / 2)}+E_{0} e^{-p(x+d / 2)} & d / 2>x>-d / 2 \\ F_{0} e^{k(x+d / 2)}+G_{0} e^{-k(x+h+d / 2)} & -d / 2>x>-h-d / 2 \\ H_{0} e^{p(x+h+d / 2)} & -h-d / 2>x,\end{cases}
$$

in which $k=\sqrt{\beta^{2}-k_{0}^{2} \varepsilon_{1}}$ and $p=\sqrt{\beta^{2}-k_{0}^{2} \varepsilon_{2}}$ are the transverse propagation constants (along the $X$ axis) of SPPs in dielectric $\left(\varepsilon_{1}\right)$ and metal $\left(\varepsilon_{2}\right)$, respectively, and $\beta$ and $k_{0}$ are that of SPPs in a waveguide (along the $Z$ direction) and incident light in vacuum, respectively. $A_{0}, B_{0}, C_{0}, D_{0}, E_{0}, F_{0}, G_{0}$, and $H_{0}$ are the mode amplitudes of SPPs in metal and dielectric of the guides, respectively, on different interfaces. By applying the boundary condition that $H_{y}$ must be continuous on the interfaces, Eq. (1) can be reduced to: 

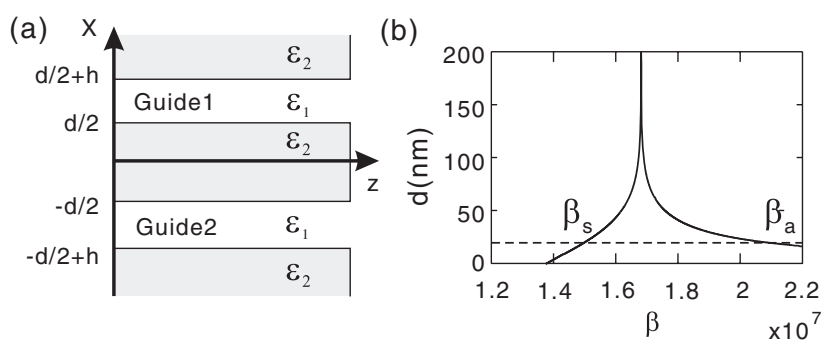

FIG. 1. (a) Scheme of evanescently coupled metal waveguides. (b) Dependence of the propagation constant $\beta$ of SPPs on the thickness of metal film $d$, as the incident wavelength $\lambda=$ $632.8 \mathrm{~nm}$ and the waveguides width $h=30 \mathrm{~nm}$.

$$
\left\{\begin{array}{l}
A_{0}=B_{0}+C_{0} e^{-k h} \\
B_{0} e^{-k h}+C_{0}=D_{0}+E_{0} e^{-p d} \\
D_{0} e^{-p d}+E_{0}=F_{0}+G_{0} e^{-k h} \\
F_{0} e^{-k h}+G_{0}=H_{0} .
\end{array}\right.
$$

A similar $E_{z}$ distribution can be obtained by applying Maxwell equations on Eq. (1). Then, by solving the equations of $E_{z}$ and $H_{y}$, we get the dispersion relation of SPPs [24]:

$$
\frac{(1-b)}{(1+b)}= \pm e^{d p}
$$

in which $b=\left[\varepsilon_{2} k+\varepsilon_{1} p-\left(\varepsilon_{2} k-\varepsilon_{1} p\right) e^{-2 k h}\right] \varepsilon_{2} k /$ $\left[\varepsilon_{2} k+\varepsilon_{1} p+\left(\varepsilon_{2} k-\varepsilon_{1} p\right) e^{-2 k h}\right] \varepsilon_{1} p$. The relationship between mode amplitudes $D_{0}$ and $E_{0}$ in metal at $x=d / 2$ and $x=-d / 2$, respectively, is

$$
\frac{D_{0}}{E_{0}}=\frac{1-b}{1+b} e^{-P d}=\frac{1+b}{1-b} e^{P d},
$$

which can be used to denote the relative amplitudes and phases of $H_{y}$ in guides 1 and 2, respectively. Figure 1(b) shows the dependence of propagation constant $\beta$ of SPP modes on $d$, as $h=30 \mathrm{~nm}, d=20 \mathrm{~nm}$, and $\varepsilon_{1}=1$. The horizontal dashed line in Fig. 1(b) corresponds to the situation of $d=20 \mathrm{~nm}$. We can see from the figure that, for a certain guide width $d$, Eq. (3) has two solutions $\beta_{s}$ and $\beta_{a}$. By substituting these two solutions into Eq. (4), we will find that when $\beta=\beta_{s}$, we have $D_{0} / E_{0}=1$, which indicates that the modes in the two waveguides are symmetric, while when $\beta=\beta_{a}$, we will get $D_{0} / E_{0}=-1$, indicating that the modes in the two waveguides are antisymmetric. So, $\beta_{s}$ and $\beta_{a}$ are the symmetry and antisymmetry mode's propagation constant.

From the coupled-wave theory, we know that the field distribution of propagating SPPs in MWGAs satisfies the following differential equation [21]:

$$
i \frac{d a_{n}(z)}{d z}+C\left[a_{n-1}(z)+a_{n+1}(z)\right]=0,
$$

where $a_{n}(z)$ represents the amplitude of magnetic field $H$ in the $n$th guide, and $C=\left(\beta_{s}-\beta_{a}\right) / 2$ is the coupling coefficient [25]. The dispersion relation of SPPs in MWGAs is [26]

$$
k_{z}=\beta+2 C \cos \left(k_{x} D\right),
$$

where $\beta=\left(\beta_{s}+\beta_{a}\right) / 2, k_{x}$ and $k_{z}$ are the $x, z$ component of wave vector $k$ of SPPs, and $D=d+h$ is the waveguide period, which determines the size of Brillouin zone and the period of the periodic dispersion. From Fig. 1(b), we see that $\beta_{a}>\beta_{s}$ in MWGAs, which produces a negative coupling coefficient $C$, indicating an anomalous coupling compared with common dielectric waveguide arrays, in which the coupling coefficient is generally positive. From Eq. (6), we get the dispersion relation [Fig. 2(b)] of SPPs passing through the MWGAs schematically shown in Fig. 2(a). One can see that, within the first Brillouin zone $\left(-\pi<k_{x} D<\pi\right)$, the dispersion of SPPs in MWGAs is a hyperbolic curve and, hence, is capable of realizing NR with $k \cdot V_{g}>0$ [27]. From the definition of group velocity $v_{g}=\frac{d \omega}{d k}$, one knows that $v_{g}$ of SPPs must lie normal to the isofrequency contour [28]. Then we can see that, within $-\pi / 2<k_{x} D<\pi / 2$, the group velocity of SPPs shows NR while the phase velocity (wave vector) undergoes positive refraction $[9,29]$.

To further demonstrate the analytical results, we numerically simulate the propagation behavior of a light beam passing through the interface between a homogenous dielectric (air) and a MWGA [Fig. 2(a)] constructed with silver using the FDTD method. The relative permittivity of $\mathrm{Ag}$ is taken to be the measured value of $\varepsilon_{2}=-15.7+$ $0.94 j$ at the excited light wavelength $(\lambda=632.8 \mathrm{~nm})$ [30]. The dielectric layer in the guide region is taken to be air $\left(\varepsilon_{1}=1\right)$. The thicknesses of the air layer is $30 \mathrm{~nm}$ and that of $\mathrm{Ag}$ film is $20 \mathrm{~nm}$ (thinner than the skin depth of $\mathrm{Ag}$ of $25 \mathrm{~nm}$ [31]), so that the period of MWGAs $D=50 \mathrm{~nm}$.
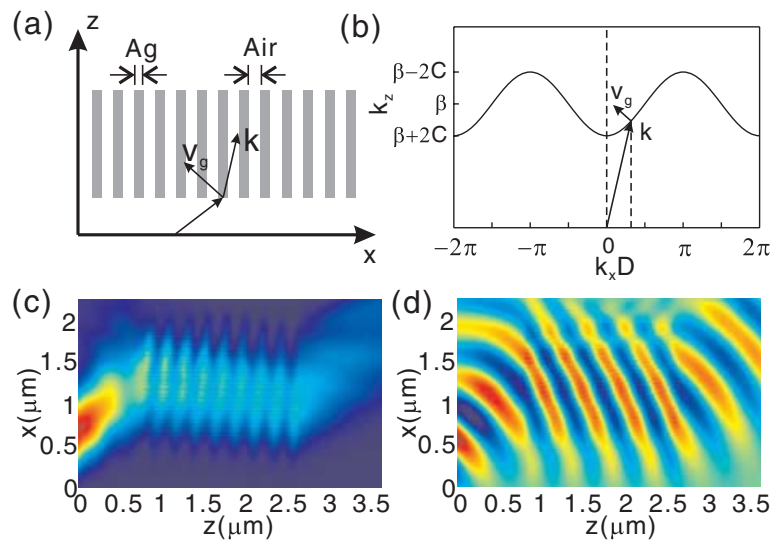

FIG. 2 (color online). (a) Scheme of a $1.8 \mu \mathrm{m}$-long MWGA composed of 40 pieces of waveguides with a $20 \mathrm{~nm}$ - and $30 \mathrm{~nm}$ thick Ag film and air layer, respectively, and a refraction diagram. (b) Dispersion relation of SPPs in the MWGAs. (c) $\left|H_{y}\right|^{2}$ distribution and (d) phase wave front of SPPs in MWGAs as a light beam is incident into a MWGA. 
When a TM-polarized Gaussian light beam with the full width at half maximum (FWHM) of $690 \mathrm{~nm}$ is incident into the MWGAs with a $40^{\circ}$ angle, the simulated distribution is presented in Fig. 2(c). One can see that the incident and refracted beams are indeed in the same side of the normal of the interface, meaning that an NR phenomenon appears. From the phase plot of SPPs in MWGAs [Fig. 2(d)], however, one can see that, as indicated in the dispersion relation of Fig. 2(b), the phase velocity of SPP modes in MWGAs shows positive refraction.

For the purpose of getting AANR with MWGAs, one has to satisfy the condition $\left|k_{x} D\right|<\pi / 2$ [Fig. 2(b)]. In the above case of waveguide period $D=50 \mathrm{~nm}$, we see that AANR can be achieved only if the incident light wavelength is longer than $200 \mathrm{~nm}(|2 \pi / \lambda \cdot D|<\pi / 2)$. This implies that MWGAs can show AANR in a large range of frequency bandwidth from visible to near-infrared regions $(\lambda>200 \mathrm{~nm}$ in our present case) by modulating the geometry of MWGAs.

From the slope of the isofrequency contour, we can determine the refraction angle $\theta$ from:

$$
\tan (\theta)=\frac{d k_{z}}{d k_{x}}=-2 C D \sin \left(k_{x} D\right)
$$

in which $k_{x}=k_{0} \sin (\varphi)$, and $\varphi$ is the incident angle. It is straightforward to get the effective refractive index of MWGAs by Snell's law:

$$
n_{\text {eff }}=\sin (\varphi) / \sin (\theta) .
$$

Figure 3 shows the relation between $n_{\text {eff }}$ of MWGAs in Fig. 2(a) and $\varphi$ at $\lambda=632.8 \mathrm{~nm}$. We find that, except for a small variation with the change of incident angle, $n_{\text {eff }}$ is always negative, indicating again that AANR can be realized.

Reproducible realization of nanometer-sized air gaps between $\mathrm{Ag}$ films for constructing MWGAs by current lithography-based technologies is difficult. However, deposition of nanometer-thick metal and dielectric films is sufficiently feasible with conventional techniques. There-

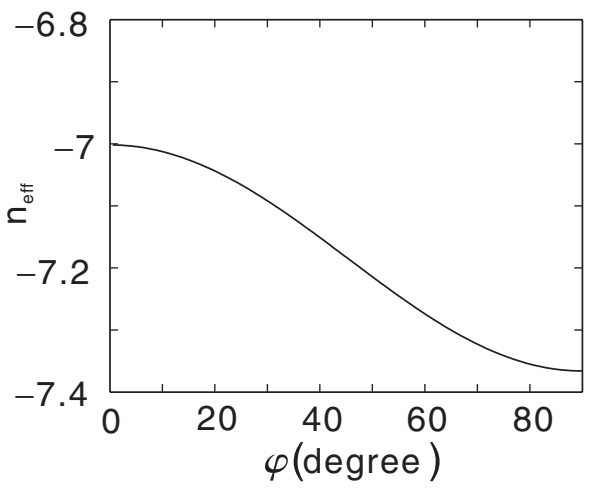

FIG. 3. Dependence of the effective refractive index of MWGAs on the incident angle $\varphi$ of the light beam at $\lambda=$ $632.8 \mathrm{~nm}$. fore, in the following, we introduce nanometer-thick dielectric interlayers to connect the MWGAs, which should facilitate the realization of MWGAs and the demonstration of novel phenomena associated with the AANR in broadband from visible to near-infrared ranges. Figure 4(a) schematically shows the setup for the imaging of MWGAs composed of 40 pieces of guides. The geometrical parameters of the structure are selected to be $h=$ $40 \mathrm{~nm}, d=10 \mathrm{~nm}$, and $\varepsilon_{1}=2.25\left(\mathrm{SiO}_{2}\right)$ so as to make $n_{\text {eff }} \sim-1$ for the MWGAs. A line source $(\lambda=632.8 \mathrm{~nm})$ is placed $400 \mathrm{~nm}$ away from the interface between air and MWGAs. Figures 4(b) and 4(c) present the $\left|H_{y}\right|^{2}$ distribution and the phase wave front of $H_{y}$, respectively, as SPPs pass through a $1.15 \mu \mathrm{m}$-long MWGA. We get an image on the other side of MWGAs with a FWHM of $280 \mathrm{~nm}$ [Fig. 4(d)], which is smaller than $\lambda / 2$ and thus achieving superdiffraction limit imaging. This can further be confirmed by observing the evolution of SPPs passing through the MWGAs. Figure 5 shows the normalized intensity evolution of a light wave passing through the free space, a dielectric lens, and a MWGA, respectively, within 30000 time steps. We can see that, after 5000 time steps, light wave possesses a strong oscillation in the image plane of MWGAs. Further observation on the light wave at the immediate exit facet of MWGAs shows stronger oscillations than that in its image plane, indicating that MWGAs can collect some evanescent components of light wave for imaging with a resolution beyond the diffraction limit [32]. With the well developed scanning near-field optical microscopy, one is capable of experimentally characterizing the above AANR effect and imaging properties of propagation SPPs through the MWGAs.

For any application that employs SPPs, metallic loss is one of the major concerns. For example, the propagation
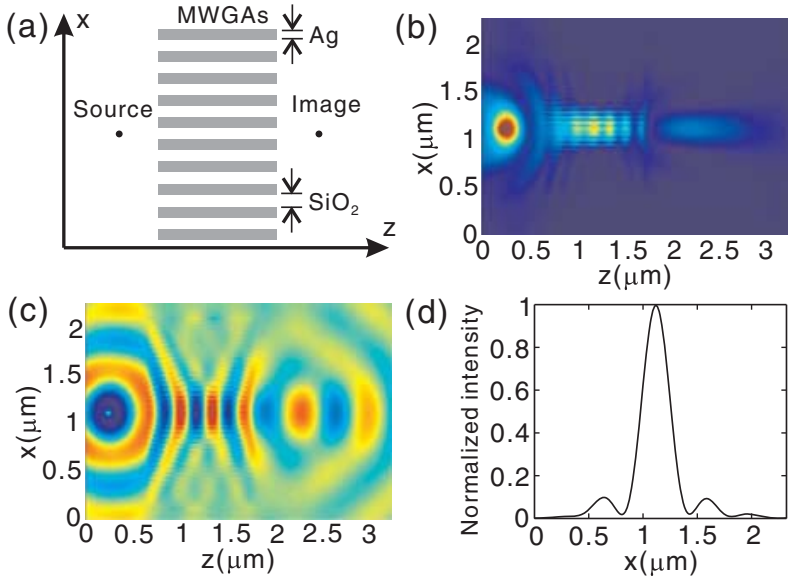

FIG. 4 (color online). (a) Setup for the imaging of MWGAs. (b) $\left|H_{y}\right|^{2}$ distribution as SPPs pass through a $1.15 \mu \mathrm{m}$-long MWGA composed of 40 pieces of metal waveguides with a $10 \mathrm{~nm}$ - and $40 \mathrm{~nm}$-thick $\mathrm{Ag}$ film and dielectric layer $\left(\mathrm{SiO}_{2}, \varepsilon_{1}=\right.$ 2.25), respectively. (c) Phase wave front of $H_{y}$. (d) $\left|H_{y}\right|^{2}$ distribution in the image plane. 


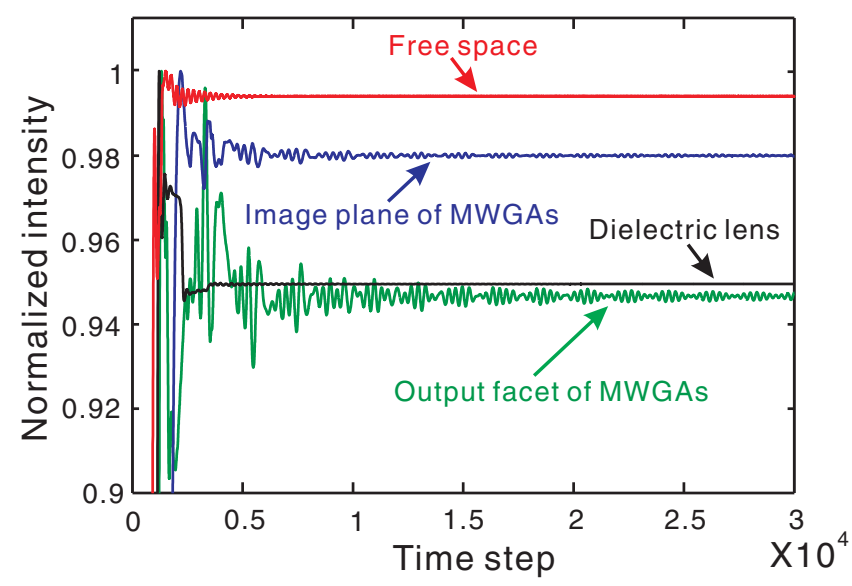

FIG. 5 (color online). Evolution of the normalized $\left|H_{y}\right|^{2}$ of a light wave passing through a free space, a dielectric lens, and a MWGA, respectively, at the image planes of the lens and MWGAs and the immediate output facet of the MWGAs, respectively.

loss of SPPs in MWGAs may affect their imaging effect. However, as shown above (Fig. 4), even in the case of using realistic metal Ag, the MWGAs can still realize functional imaging. By operating at low temperature [33] or by introducing a gain medium [34] to quench the effect of losses, the imaging effect of MWGAs should be further improved.

In conclusion, we showed that MWGAs can realize allangle broadband negative refraction in the optical range from the visible to the infrared region by the coupled-wave theory, and the analytical results are confirmed by FDTD numerical simulation. The negative coupling constant resulted from the anomalous coupling of guided SPPs is attributed to the negative refraction. Our modes provide a robust way to construct AANR materials operating in a wide frequency band from near-infrared to visible ranges.

X.F. and G.P.W. acknowledge financial support from Ministry of Education of China (No. NCET-04-0678) and NSFC (Grant No. 10574101). J.C. W. L. and C. T.C. are supported by HK-RGC through Grant No. 600403.

*Corresponding author.

Electronic address: kp_wang@hotmail.com

[1] V. G. Veselago, Sov. Phys. Usp. 10, 509 (1968).

[2] J. B. Pendry, Phys. Rev. Lett. 85, 3966 (2000).

[3] J. B. Pendry and D. R. Smith, Phys. Today 57, No. 6, 37 (2004).

[4] D. R. Smith, W. J. Padilla, D. C. Vier, S. C. Nemat-Nasser, and S. Schultz, Phys. Rev. Lett. 84, 4184 (2000).
[5] T. Koschny, M. Kafesaki, E. N. Economou, and C. M. Soukoulis, Phys. Rev. Lett. 93, 107402 (2004).

[6] D. R. Smith, J. B. Pendry, and M. C. K. Wiltshire, Science 305, 788 (2004).

[7] S. Zhang, W. Fan, N. C. Panoiu, K. J. Malloy, R. M. Osgood, and S. R. J. Brueck, Phys. Rev. Lett. 95, 137404 (2005).

[8] M. Notomi, Phys. Rev. B 62, 10696 (2000).

[9] C. Luo, S. G. Johnson, J. D. Joannopoulos, and J. B. Pendry, Phys. Rev. B 65, 201104(R) (2002).

[10] X. Zhang and L. Li, Appl. Phys. Lett. 86, 121103 (2005).

[11] E. Cubukcu, K. Aydin, E. Ozbay, S. Foteinopoulou, and C. M. Soukoulis, Nature (London) 423, 604 (2003).

[12] P. V. Parimi, W. T. Lu, P. Vodo, and S. Sridhar, Nature (London) 426, 404 (2003).

[13] X. Ao and S. He, Appl. Phys. Lett. 87, 101112 (2005).

[14] A. Berrier, M. Mulot, M. Swillo, M. Qiu, L. Thylén, A. Talneau, and S. Anand, Phys. Rev. Lett. 93, 073902 (2004).

[15] I. Bulu, H. Caglayan, and E. Ozbay, Phys. Rev. B 72, 045124 (2005).

[16] R. A. Shelby, D. R. Smith, and S. Schultz, Science 292, 77 (2001).

[17] A. A. Houck, J. B. Brock, and I. L. Chuang, Phys. Rev. Lett. 90, 137401 (2003).

[18] Z. Feng, X. Zhang, Y. Wang, Z. Y. Li, B. Cheng, and D. Z. Zhang, Phys. Rev. Lett. 94, 247402 (2005).

[19] X. Ao and S. He, Opt. Lett. 29, 2542 (2004).

[20] C. R. Rosberg, D. N. Neshev, A. A. Sukhorukov, Y.S. Kivshar, and W. Krolikowski, Opt. Lett. 30, 2293 (2005).

[21] T. Pertsch, T. Zentgraf, U. Peschel, A. Brauer, and F. Lederer, Phys. Rev. Lett. 88, 093901 (2002).

[22] B. Wang and G. P. Wang, Opt. Lett. 29, 1992 (2004).

[23] X. Fan and G. P. Wang, Opt. Lett. 31, 1322 (2006).

[24] E. N. Economous, Phys. Rev. 182, 539 (1969).

[25] A. Locatelli, M. Conforti, D. Modotto, and C. De Angelis, Opt. Lett. 30, 2894 (2005).

[26] H. S. Eisenberg, Y. Silberberg, R. Morandotti, and J. S. Aitchison, Phys. Rev. Lett. 85, 1863 (2000).

[27] S. Foteinopoulou and C. M. Soukoulis, Phys. Rev. B 67, 235107 (2003).

[28] D. R. Smith and D. Schurig, Phys. Rev. Lett. 90, 077405 (2003).

[29] D. R. Smith, D. Schurig, and J. B. Pendry, Appl. Phys. Lett. 81, 2713 (2002).

[30] Handbook of Optical Constants of Solids, edited by E. D. Palik (Academic, New York, 1985).

[31] H. Raether, Surface Plasmon (Springer-Verlag, Berlin, 1988).

[32] L. Zhou and C. T. Chan, Appl. Phys. Lett. 86, 101104 (2005).

[33] A. Karalis, E. Lidorikis, M. Ibanescu, J. D. Joannopoulos, and M. Solja, Phys. Rev. Lett. 95, 063901 (2005).

[34] M. P. Nezhad, K. Tetz, and Y. Fainman, Opt. Express 12, 4072 (2004). 